\title{
Korean Red Ginseng Improves Blood Pressure Stability in Patients with Intradialytic Hypotension
}

\section{I-Ju Chen, ${ }^{1,2}$ Ming-Yang Chang, ${ }^{3}$ Sheng-Lin Chiao, ${ }^{1}$ Jiun-Liang Chen, ${ }^{1,4}$ Chun-Chen Yu, ${ }^{3}$ Sien-Hung Yang, ${ }^{1,4}$ Ju-Mei Liu, ${ }^{5}$ Cheng-Chieh Hung, ${ }^{3}$ Rong-Chi Yang, ${ }^{4}$ Hui-Chi Chang, ${ }^{6}$ Chung-Hua Hsu, ${ }^{2}$ and Ji-Tseng Fang ${ }^{3}$}

\author{
${ }^{1}$ Center for Traditional Chinese Medicine, Chang Gung Memorial Hospital, Taoyuan 333, Taiwan \\ ${ }^{2}$ Branch of Chinese Medicine and Linsen, Taipei City Hospital, Taipei 103, Taiwan \\ ${ }^{3}$ Department of Nephrology, Kidney Research Center, Chang Gung Memorial Hospital, \\ Chang Gung University College of Medicine, 5 Fu-Shin Street, Kueishan, Taoyuan 333, Taiwan \\ ${ }^{4}$ Chang Gung University College of Medicine, Taoyuan 333, Taiwan \\ ${ }^{5}$ Department of Internal Medicine, Taipei Medical University Hospital, Taipei 110, Taiwan \\ ${ }^{6}$ Departments of Nuclear Medicine, Chang Gung Memorial Hospital, Taoyuan 333, Taiwan
}

Correspondence should be addressed to Ji-Tseng Fang, fangjits@ms4.hinet.net

Received 5 November 2011; Revised 21 January 2012; Accepted 21 January 2012

Academic Editor: Myeong Soo Lee

Copyright (C) 2012 I-Ju Chen et al. This is an open access article distributed under the Creative Commons Attribution License, which permits unrestricted use, distribution, and reproduction in any medium, provided the original work is properly cited.

\begin{abstract}
Introduction. Intradialytic hypotension (IDH) is a common complication during hemodialysis which may increase mortality risks. Low dose of Korean red ginseng (KRG) has been reported to increase blood pressure. Whether KRG can improve hemodynamic stability during hemodialysis has not been examined. Methods. The 8-week study consisted of two phases: observation phase and active treatment phase. According to prehemodialysis blood pressure (BP), 38 patients with IDH were divided into group $\mathrm{A}(\mathrm{BP} \geq 140 / 90 \mathrm{mmHg}, n=18)$ and group $\mathrm{B}(\mathrm{BP}<140 / 90 \mathrm{mmHg}, n=20)$. Patients were instructed to chew 3.5 gm $\mathrm{KRG}$ slices at each hemodialysis session during the 4-week treatment phase. Blood pressure changes, number of sessions disturbed by symptomatic IDH, plasma levels of vasoconstrictors, blood biochemistry, and adverse effects were recorded. Results. KRG significantly reduced the degree of blood pressure drop during hemodialysis $(P<0.05)$ and the frequency of symptomatic IDH $(P<0.05)$. More activation of vasoconstrictors (endothelin-1 and angiotensin II) during hemodialysis was found. The postdialytic levels of endothelin-1 and angiotensin II increased significantly $(P<0.01)$. Conclusion. Chewing KRG renders IDH patients better resistance to acute BP reduction during hemodialysis via activation of vasoconstrictors. Our results suggest that KRG could be an adjuvant treatment for IDH.
\end{abstract}

\section{Introduction}

Intradialytic hypotension (IDH) is a common complication which occurs in $20-30 \%$ of all dialysis treatments [1]. It has a negative impact on quality of life due to associated symptoms such as nausea, vomiting, dizziness, and cramps. IDH may cause premature discontinuation of hemodialysis that may lead to chronic underdialysis and higher mortality risks $[1,2]$.

The mechanism responsible for IDH is an inappropriate response of cardiovascular and neurohormonal systems to the acute plasma volume removal during dialysis [3-5].
Hemodialysis prescriptions include ultrafiltration amount, dialysate composition, and solution temperature may affect the frequency of IDH [6-8]. Older age, ischemic heart disease, diabetes, and autonomic neuropathy increase the risk of developing IDH [9-11]. Common interventions to prevent IDH include adaptation of dialysis prescriptions, avoidance of food during dialysis, and administration of vasoconstrictor agents (e.g., midorine, adenosine antagonist) $[1,6,12-14]$.

Panax ginseng, a naturally occurring compound that has been used for several thousand years in the orient [15], is one of the most popular herbs in the world due to its therapeutic 
effects on modulating immune and cardiovascular functions [16]. Previous studies on Panax ginseng have confirmed its effect on regulating blood pressure [15]. Interestingly, Panax ginseng at low-doses can elevate blood pressure, while highdose Panax ginseng has hypotensive effect in healthy subjects [17-19]. However, the potential therapeutic effect of Panax ginseng in patients with IDH has not been examined.

Korean red ginseng (KRG), a steamed form of Panax ginseng with preserved major constituents, has been shown to possess more biological activity than Panax ginseng [20-22]. Here we conducted a prospective study to evaluate the effects of KRG on the occurrence of symptomatic IDH during hemodialysis. We also examined the changes of endothelin-1 (ET-1), plasma renin activity (PRA), angiotensin II (AngII), and nitric oxide (NO) products during hemodialysis with and without oral KRG administration.

\section{Material and Methods}

2.1. Participants. The study was conducted at the hemodialysis center in Taoyuan Chang Gung Memorial Hospital (TCGMH), Taiwan. The study was approved by Chang Gung Memorial Hospital Ethical Review Committees. Each patient signed informed consent before enrollment. Patients aged from 20- to 80-year-old on thrice-weekly hemodialysis, with a treatment time of at least 180 minutes, and had at least three symptomatic episodes of IDH in the 30 days preceding enrollment, were enrolled in the study. IDH was defined as a decrease in systolic blood pressure $(\geq 20 \mathrm{mmHg})$ or a decrease in mean arterial pressure $(\geq 10 \mathrm{mmHg})$ accompanied by symptoms (dizziness, cramps, or fatigue, etc.), according to Kidney Disease Outcomes Quality Initiative Clinical Practice Guidelines for Cardiovascular Disease in Dialysis Patients [23]. The exclusion criteria included pregnancy or breast feeding, active infectious disease, current intake of antihypotensive medication or warfarin, and frequent changes of dry body weight $(> \pm 1 \%$ during the screening phase) and severe medical conditions included liver cirrhosis, heart failure, autoimmune disease, and cancer. Of the 768 patients receiving chronic dialysis in our hospital, 74 patients were eligible for this study; 46 patients were enrolled, while 28 patients declined to participate (Figure 1).

2.2. Study Design. The prospective study was designed to evaluate the pre- and posttreatment differences, and each patient served as his/her own control. The 8-week study consisted of two phases: an observation phase (phase I) and an active treatment phase (phase II). Each phase was composed of twelve consecutive HD treatments (4 weeks). Patients were given standard treatment for IDH during phase I. These enrolled patients were divided into two groups according to their prehemodialysis blood pressure. Patients with average prehemodialysis blood pressure more than $140 \mathrm{mmHg}$ systolic or $90 \mathrm{mmHg}$ diastolic during phase I were clustered to group $\mathrm{A}$, while patients with normal or low prehemodialysis blood pressure to group $\mathrm{B}$.

2.3. Treatment. During the active treatment phase (phase II), patients were instructed to chew $3.5 \mathrm{gm}$ KRG slices at each hemodialysis session. Each slice of KRG was put into the mouth until melted, then chewed and swallowed. Patients in group A (hypertensive at baseline) were given KRG slices 60 minutes after the onset of hemodialysis to prevent late onset of hypotension after ultrafiltration. In contrast, patients in group B (normotensive or hypotensive at baseline) were given KRG slices 30 minutes before hemodialysis to prevent early onset of hypotension [24]. Cheong-Kwan-Jang Korean red ginseng (Korea Ginseng Corporation; Korea) was used in this study. The active constituents of KRG are ginsenosides including Rb1 (1.96\%), Rb2 (2.18\%), Rc (1.47\%), Rd (0.72\%), Re (1.11\%), Rf (0.24\%), Rg1 (0.49\%), Rg2 (0.13\%), Rg3 (0.12\%), Rh1 (0.12\%), and Rh2 (0.003\%) [25]. Routine dialysis prescriptions including dialyzer types, dialysate compositions, dialysate temperature, dialysis frequencies, treatment time, and antihypertensive medications were maintained constantly throughout two study phases.

2.4. Outcome Parameters. Arterial blood pressure was measured with an electronic digital sphygmomanometer every 60 minutes from the beginning to the end of each dialysis session. In the event of IDH, the blood pressure was checked every 10 minutes. Prehemodialysis (pre-HD), posthemodialysis (post-HD), and intradialytic lowest (nadir) blood pressure at each dialysis session were recorded. The difference of SBP, DBP, and MAP between prehemodialysis and nadir, and between prehemodialysis and posthemodialysis, were calculated. The number of sessions disturbed by symptomatic IDH was defined as hypotensive episodes that require medical intervention including transient reduction or premature stop of ultrafiltration, infusion of isotonic or hypertonic saline, or glucose solution. These parameters during each study phase were recorded by the nurses at the hemodialysis center. Any adverse effects that might be related to KRG were recorded.

2.5. Assays. In the last dialysis sessions of each study phase, plasma levels of ET-1, PRA, AngII, and NO products were checked before and after dialysis. Blood samples were collected with prechilled polypropylene tubes containing $1 \mathrm{mg} / \mathrm{mL}$ of K2-EDTA and $500 \mathrm{KIU} / \mathrm{mL}$ aprotinin (Sigma). Plasma was separated by centrifugation at $3000 \mathrm{rpm}$ for 15 minutes at $4^{\circ} \mathrm{C}$ and stored at $-70^{\circ} \mathrm{C}$ until assayed. Specific antibodies and radioimmunoassay kits were used to assay PRA (DiaSorin, MN, USA), AngII (Phoenix Pharmaceuticals Inc. USA), and ET-1 (Peninsula Laboratories, LLC, USA). The final products of NO metabolism were examined using an assay for detecting the plasma levels of the nitrate+nitrite (NT) (Cayman Chemical Company, Ann Arbor). Hematocrit, albumin, electrolytes, and alanine aminotransferase were monitored at the beginning and end of the study by autoanalyzer.

2.6. Statistical Analyses. All numerical values are expressed as mean \pm standard deviation (SD). Baseline variables are compared with the $\chi^{2}$ test for dichotomous variables. The MannWhitney $U$ test is applied to compare intergroup differences. The Wilcoxon signed-rank test is used for intragroup 


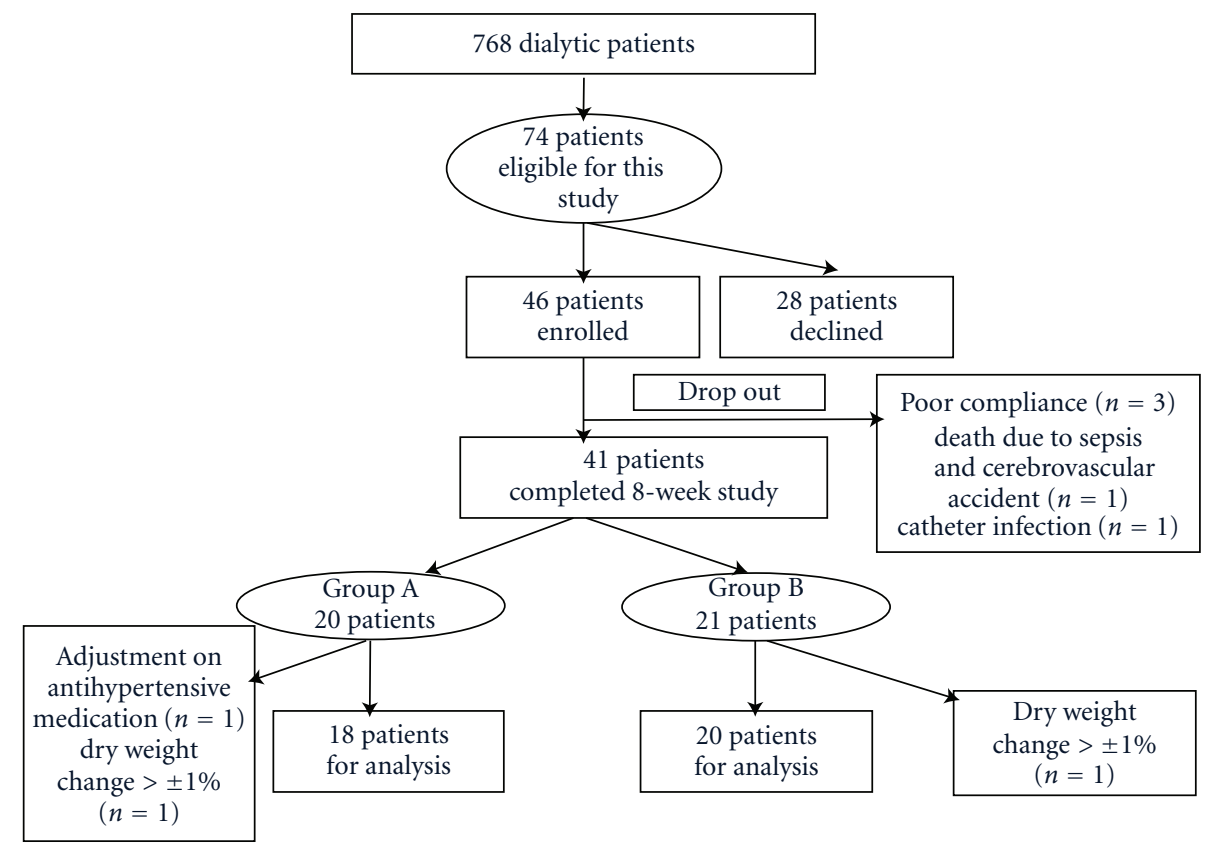

FIgURE 1: Flow chart of patient recruitment. Patients aged from 20 to 80 year old on thrice-weekly hemodialysis, with a treatment time of at least 180 minutes and had at least three symptomatic episodes of intradialytic hypotension (IDH) in the 30 days preceding enrollment, were enrolled in the study.

TABLE 1: Baseline characteristics of study patients.

\begin{tabular}{|c|c|c|c|c|}
\hline & Total $(n=38)$ & Group A (HTN) $(n=18)$ & Group B (Non-HTN) $(n=20)$ & $P$ value \\
\hline Age (year) & $52.6 \pm 12.4$ & $56.2 \pm 12.7$ & $49.3 \pm 11.5$ & 0.083 \\
\hline Sex (\% Female) & $25(65.8 \%)$ & $11(61.1 \%)$ & $14(70 \%)$ & 0.734 \\
\hline Dry BW (kg) & $60.7 \pm 16.6$ & $62.5 \pm 15.0$ & $59.0 \pm 18.1$ & 0.515 \\
\hline BMI & $23.6 \pm 5.2$ & $24.1 \pm 4.2$ & $23.1 \pm 6$ & 0.579 \\
\hline Time on dialysis (months) & $93.0 \pm 61.9$ & $67.9 \pm 48.7$ & $115.5 \pm 64.9$ & $0.016^{*}$ \\
\hline \multicolumn{5}{|l|}{ Comorbidities } \\
\hline $\mathrm{DM}$ & $15(39.5 \%)$ & $13(72.2 \%)$ & $2(10 \%)$ & $<0.001^{* *}$ \\
\hline HTN history & $26(68.4 \%)$ & $18(100 \%)$ & $8(40 \%)$ & $<0.001^{* *}$ \\
\hline Previous MI & $1(2.6 \%)$ & $1(5.6 \%)$ & $0(\%)$ & 0.474 \\
\hline Angina history & $5(13.2 \%)$ & $3(16.7 \%)$ & $2(10 \%)$ & 0.653 \\
\hline
\end{tabular}

Values are expressed as means \pm SD or number (\%); BMI, body mass index; BW, body weight; DM, diabetes mellitus; HTN, hypertension; MI, myocardial infarction; ${ }^{*} P<0.05 ;{ }^{* *} P<0.01$.

comparison between phase I and phase II. A two-tailed $P<$ 0.05 is considered statistically significant. Statistical analysis is performed with analytical Software SPSS 12.0 version.

\section{Results}

3.1. Patient Characteristics. Of the 46 enrolled patients, 21 patients were grouped into group $\mathrm{A}$ and 25 patients to group B according to prehemodialysis blood pressure in the observation phase (phase I) (Figure 1). In total, 41 patients completed the KRG treatment phase (phase II), and five patients dropped out of the study prematurely due to poor compliance ( $n=1$ in group A; $n=2$ in group B), catheter infection ( $n=1$ in group $\mathrm{B})$, and death due to sepsis and cerebrovascular accident $(n=1$ in group $\mathrm{A})$ which was not directly related to KRG use. For the statistical analyses, three patients were excluded due to adjustment of antihypertensive medications ( $n=1$ in group A) and changes of dry weight exceeding $\pm 1 \%(n=2$ in group B). Finally, 18 patients in the group A and 20 patients in the group B were included for analysis.

Baseline patient characteristics of the 38 patients of the analysis population are listed in Table 1 . The mean age was $53 \pm 12$ years; $25(65.8 \%)$ were females and $13(34.2 \%)$ were males. Compared to patients in group B (normal or low prehemodialysis BP), patients in group A (high prehemodialysis BP) had a significantly shorter average time on maintenance dialysis ( $68 \pm 49$ versus $116 \pm 65$ months, $P=0.016)$ and a higher percentage of diabetes mellitus (72.2\% versus $10 \%, P<0.001)$. 


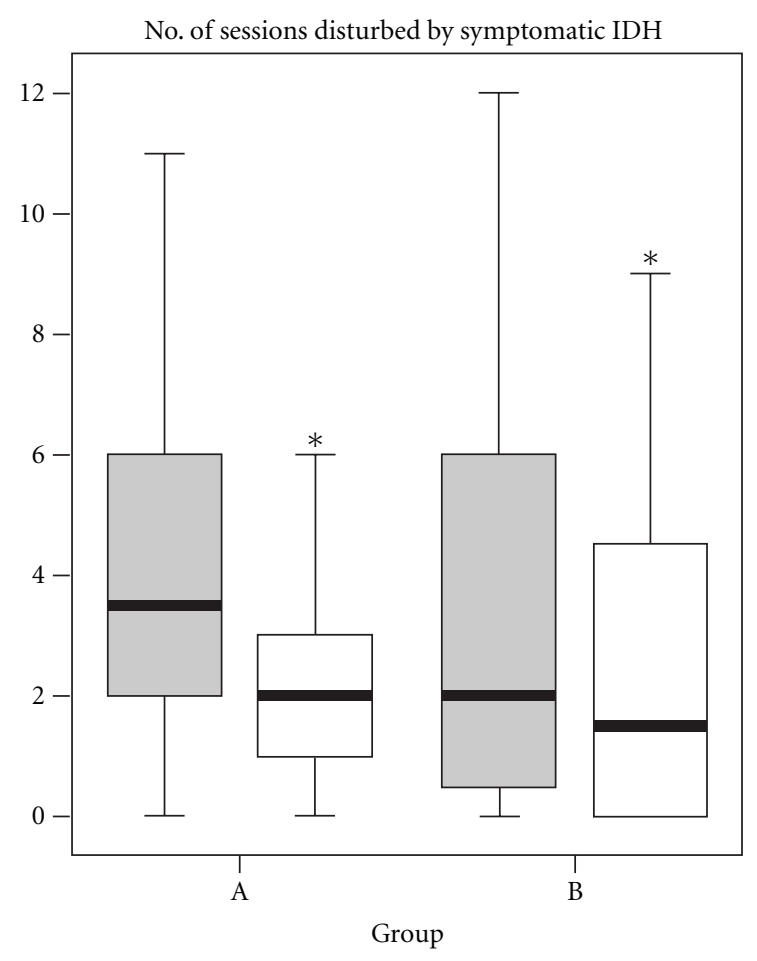

Phase I (-KRG)

Phase II (+KRG)

FIGURE 2: Comparison of the frequency of intradialytic hypotension (IDH) with and without Korean red ginseng (KRG) treatment. Phase I is the control phase (grey boxes). Phase II is the KRG treatment phase (white boxes). The number of sessions disturbed by symptomatic IDH reduced significantly after KRG treatment in both group A (hypertensive at baseline, $n=18, P=0.016$ ) and group B (normotensive or hypotensive at baseline, $n=20$, $P=0.035$ ). Data are shown as box and whisker plots. Horizontal lines represent median values. The boxes encompass the first and 3 rd quartile of the included data. The bars give the $95 \%$ confidence interval of the values. ${ }^{*} P<0.05$.

3.2. Effect of KRG on Symptomatic IDH. We examined the clinical effects of KRG on symptomatic IDH. The number of sessions disturbed by symptomatic IDH reduced significantly from $3.9 \pm 3.1$ times (per 12 times) to $2.7 \pm 3.1$ times in group $\mathrm{A}(P=0.016)$ and from $3.9 \pm 3.9$ times to $2.7 \pm 3.5$ times in group $\mathrm{B}(P=0.035)$ (Figure 2$)$. The average pre- and postdialysis body weight, dry weight, and the amount of actual ultrafiltration were not significantly different between two study phases (Table 2). Thus, we could exclude the effect of altered ultrafiltration amount that might have affected the occurrence of IDH.

3.3. Effect of KRG on Blood Pressure during Hemodialysis. We looked at the effects of KRG in regulating the blood pressure changes during hemodialysis (Table 3 ). We found that the nadir SBP in group B was significantly elevated from $76.3 \pm$ $16.2 \mathrm{mmHg}$ in phase I to $79.1 \pm 15.8 \mathrm{mmHg}$ in phase II $(P=$ $0.045)$. A similar trend of increased nadir SBP was noted in group $\mathrm{A}(97.4 \pm 16.0$ in phase I versus $100.1 \pm 18.6$ in phase
II; $P=0.184)$. However, the administration of KRG did not significantly change prehemodialysis or posthemodialysis blood pressure.

Furthermore, we calculated the differences of blood pressure measured at different time points (prehemodialysis to nadir and to posthemodialysis) (Table 4). Notably, we found that patients in group B experienced less blood pressure changes during phase II $(-24.4 \pm 10.1 \mathrm{mmHg}$ of Nadir-Pre SBP in phase I to $-20.4 \pm 11.0 \mathrm{mmHg}$ in phase II, $P=0.045 ;-13.2 \pm 7.1 \mathrm{mmHg}$ of nadir-pre-DBP in phase I to $-10.4 \pm 6.6 \mathrm{mmHg}$ in phase II, $P=0.011 ;-16.9 \pm 7.1 \mathrm{mmHg}$ of nadir-pre-MAP in phase I to $-13.8 \pm 7.7 \mathrm{mmHg}$ in phase II, $P=0.015 ;-11.0 \pm 6.3 \mathrm{mmHg}$ to $-7.8 \pm 6.3 \mathrm{mmHg}$ in post-pre-DBP, $P=0.025)$. A similar trend was also noted in group A after KRG treatment $(-50.7 \pm 39.3 \mathrm{mmHg}$ of nadirpre-SBP in phase I to $-42.0 \pm 23.8 \mathrm{mmHg}$ in phase II, $P=$ $0.053 ;-40.3 \pm 39.3 \mathrm{mmHg}$ of nadir-pre-MAP in phase I to $-31.8 \pm 24.8 \mathrm{mmHg}$ in phase II, $P=0.02)$. When all patients were included for analysis, the blood pressure drop reduced significantly in the KRG-treated period $(-36.9 \pm 30.7 \mathrm{mmHg}$ of nadir-pre-SBP in phase I to $-31.0 \pm 21.3 \mathrm{mmHg}$ in phase II, $P=0.006 ;-22.4 \pm 14.5 \mathrm{mmHg}$ of nadir-pre-MAP in phase I to $-19.3 \pm 12.7 \mathrm{mmHg}$ in phase II, $P=0.004)$. These results indicate that KRG may help to keep hemodynamic stability in IDH patients.

3.4. Effect of KRG on PRA, ET-1, AngII, and NT. To investigate the action mechanism of KRG, we examined the plasma levels of PRA, ET-1, AngII, and NT (nitrite + nitrate). In the observation phase (phase I), PRA levels increased significantly after hemodialysis in group B $(P=0.004)$ but less evident in group A $(P=0.071)$. The level of ET-1 and AngII did not significantly increase despite an average ultrafiltration of $2.6 \pm 1.0 \mathrm{~kg}$ with removal of fluid in both groups $(P>0.05)$.

After four weeks of KRG treatment (phase II), the posthemodialysis PRA levels increased significantly in both groups $(P=0.005$ in group $\mathrm{A}, P<0.001$ in group B) (Figure 3). Similarly, the posthemodialysis ET-1 levels increased significantly by one- to threefold compared to the prehemodialysis levels $(P=0.035$ in group $\mathrm{A}, P=$ 0.011 in group $B)$, which is in contrast to the trend in phase I (Figure 3). The levels of AngII also significantly increased after dialysis in group $\mathrm{A}(P=0.033)$, but not in group $\mathrm{B}(P=1.000)$. A previous study has shown that NT can be removed by hemodialysis [4]. As expected, the levels of NT decreased significantly after dialysis in all groups. Nevertheless, the posthemodialysis NT levels which would cause vasodilation were significantly lower in the KRG treatment phase than those in the observatory phase $(P<0.05$ in group A) (Figure 3$)$. These results suggest that KRG treatment may improve the compensatory response mediated by various vasoconstrictors to acute volume change during hemodialysis.

Unexpectedly, we observed a two- to threefold declination of prehemodialysis ET-1 levels in both groups with KRG treatment $(1.627 \pm 1.460$ to $0.497 \pm 0.202 \mathrm{ng} / \mathrm{mL}$ in group $\mathrm{A}$, $P=0.014 ; 2.042 \pm 1.127$ to $1.023 \pm 1.190 \mathrm{ng} / \mathrm{mL}$ in group $\mathrm{B}, P=0.047)$ as shown in Figure 3 . The same holds true 
TABLE 2: Comparison of body weights and ultrafiltration rates with and without Korean red ginseng treatment.

\begin{tabular}{|c|c|c|c|c|c|c|c|c|c|}
\hline & \multicolumn{2}{|c|}{ Group A (HTN) } & \multirow{2}{*}{$P$ value } & \multicolumn{2}{|c|}{ Group B (non-HTN) } & \multirow{2}{*}{$P$ value } & \multicolumn{2}{|c|}{ All } & \multirow{2}{*}{$P$ value } \\
\hline & $\begin{array}{l}\text { Phase I } \\
(-\mathrm{KRG})\end{array}$ & $\begin{array}{l}\text { Phase II } \\
(+\mathrm{KRG})\end{array}$ & & $\begin{array}{l}\text { Phase I } \\
(-\mathrm{KRG})\end{array}$ & $\begin{array}{l}\text { Phase II } \\
(+\mathrm{KRG})\end{array}$ & & $\begin{array}{l}\text { Phase I } \\
(-\mathrm{KRG})\end{array}$ & $\begin{array}{l}\text { Phase II } \\
(+\mathrm{KRG})\end{array}$ & \\
\hline BW predialysis (Kg) & $65.3 \pm 15.5$ & $65.4 \pm 15.4$ & 0.862 & $61.6 \pm 18.8$ & $61.7 \pm 18.8$ & 0.218 & $63.4 \pm 17.2$ & $63.4 \pm 17.1$ & 0.415 \\
\hline BW postdialysis $(\mathrm{Kg})$ & $62.7 \pm 15.0$ & $62.8 \pm 15.0$ & 0.296 & $59.0 \pm 18.1$ & $58.9 \pm 18.1$ & 0.888 & $60.7 \pm 16.6$ & $60.7 \pm 16.6$ & 0.994 \\
\hline Dry BW (Kg) & $62.5 \pm 15.0$ & $62.6 \pm 15.0$ & 0.054 & $59.0 \pm 18.1$ & $59.0 \pm 18.2$ & 0.386 & $60.7 \pm 16.6$ & $60.7 \pm 16.6$ & 0.282 \\
\hline Target UF (L) & $2.6 \pm 1.0$ & $2.6 \pm 0.9$ & 0.616 & $2.7 \pm 1.0$ & $2.8 \pm 1.1$ & 0.322 & $2.6 \pm 1.0$ & $2.7 \pm 1.0$ & 0.33 \\
\hline Actual UF (L) & $2.8 \pm 1.1$ & $2.7 \pm 1.0$ & 0.850 & $2.7 \pm 1.1$ & $3.0 \pm 1.6$ & 0.198 & $2.7 \pm 1.1$ & $2.9 \pm 1.4$ & 0.338 \\
\hline$\%$ target UF & $100.0 \pm 21.5$ & $106.4 \pm 39.1$ & 0.231 & $103.8 \pm 10.4$ & $103.0 \pm 10.6$ & 0.794 & $102.0 \pm 16.5$ & $104.6 \pm 27.6$ & 0.319 \\
\hline
\end{tabular}

Values are expressed as means \pm SD; BW, body weight; KRG, Korean red ginseng; UF, ultrafiltration; \% target UF, percentage of actual UF/target UF. Phase I is the controlled phase without KRG treatment, expressed as -KRG; phase II is the KRG treatment phase, expressed as +KRG.

TABLE 3: Comparison of blood pressure measurements during hemodialysis with and without Korean red ginseng treatment.

\begin{tabular}{|c|c|c|c|c|c|c|}
\hline & \multicolumn{2}{|c|}{ Group A (HTN) } & \multirow{2}{*}{$P$ value } & \multicolumn{2}{|c|}{ Group B (non-HTN) } & \multirow{2}{*}{$P$ value } \\
\hline & Phase I (-KRG) & Phase II (+KRG) & & Phase I (-KRG) & Phase II (+KRG) & \\
\hline Pre- SBP (mmHg) & $148.1 \pm 39.3$ & $142.9 \pm 30.8$ & 0.231 & $100.8 \pm 16.9$ & $99.5 \pm 14.5$ & 0.332 \\
\hline Pre-DBP (mmHg) & $71.1 \pm 10.4$ & $71.3 \pm 13.0$ & 0.931 & $58.2 \pm 9.7$ & $56.8 \pm 8.1$ & 0.279 \\
\hline Pre-MAP (mmHg) & $96.8 \pm 18.7$ & $95.2 \pm 18.5$ & 0.327 & $72.4 \pm 11.9$ & $71.0 \pm 9.9$ & 0.218 \\
\hline Nadir SBP (mmHg) & $97.4 \pm 16.0$ & $100.1 \pm 18.6$ & 0.184 & $76.3 \pm 16.2$ & $79.1 \pm 15.8$ & $0.045^{\mathrm{a}}$ \\
\hline Nadir DBP (mmHg) & $53.9 \pm 8.4$ & $54.8 \pm 9.4$ & 0.338 & $45.0 \pm 9.8$ & $46.4 \pm 10.3$ & 0.341 \\
\hline Nadir MAP (mmHg) & $68.4 \pm 10.4$ & $69.9 \pm 11.9$ & 0.231 & $55.5 \pm 11.8$ & $57.2 \pm 12.02$ & 0.296 \\
\hline Post-SBP (mmHg) & $107.8 \pm 17.2$ & $111.1 \pm 17.2$ & 0.053 & $86.7 \pm 30.9$ & $84.8 \pm 16.4$ & 0.305 \\
\hline Post-DBP (mmHg) & $58.4 \pm 8.7$ & $59.0 \pm 8.8$ & 0.396 & $47.2 \pm 10.1$ & $49.0 \pm 10.9$ & 0.135 \\
\hline Post-MAP (mmHg) & $74.9 \pm 10.7$ & $76.4 \pm 11.1$ & 0.248 & $60.3 \pm 16.3$ & $61 \pm 12.3$ & 0.284 \\
\hline
\end{tabular}

Values are expressed as means \pm SD of the data; KRG: Korean red ginseng; pre-: prehemodialysis; post-: posthemodialysis; SBP: systolic blood pressure; DBP: diastolic blood pressure; MAP: mean arterial pressure; ${ }^{\mathrm{a}} \mathrm{P}<0.05$.

TABLE 4: Changes of blood pressure during hemodialysis with and without Korean red ginseng treatment.

\begin{tabular}{|c|c|c|c|c|c|c|c|c|c|}
\hline & \multicolumn{2}{|c|}{ Group A (HTN) } & \multirow{2}{*}{$P$ value } & \multicolumn{2}{|c|}{ Group B (non-HTN) } & \multirow{2}{*}{$P$ value } & \multicolumn{2}{|c|}{ All } & \multirow{2}{*}{$P$ value } \\
\hline & $\begin{array}{l}\text { Phase I } \\
(- \text { KRG })\end{array}$ & $\begin{array}{l}\text { Phase II } \\
(+\mathrm{KRG})\end{array}$ & & $\begin{array}{l}\text { Phase I } \\
(- \text { KRG })\end{array}$ & $\begin{array}{l}\text { Phase II } \\
(+ \text { KRG })\end{array}$ & & $\begin{array}{l}\text { Phase I } \\
(-\mathrm{KRG})\end{array}$ & $\begin{array}{l}\text { Phase II } \\
(+ \text { KRG })\end{array}$ & \\
\hline$\Delta$ Nadir-pre-SBP (mmHg) & $-50.7 \pm 39.3$ & $-42.9 \pm 23.8$ & 0.053 & $-24.4 \pm 10.1$ & $-20.4 \pm 11.0$ & $0.045^{*}$ & $-36.9 \pm 30.7$ & $-31.0 \pm 21.3$ & $0.006^{* *}$ \\
\hline$\Delta$ Nadir-pre-DBP (mmHg) & $-17.3 \pm 8.8$ & $-16.6 \pm 10.0$ & 0.446 & $-13.2 \pm 6.2$ & $-10.4 \pm 6.6$ & $0.011^{*}$ & $-15.1 \pm 7.7$ & $-13.3 \pm 8.8$ & $0.020^{*}$ \\
\hline$\Delta$ Nadir-pre-MAP (mmHg) & $-28.4 \pm 18.0$ & $-25.4 \pm 14.4$ & 0.078 & $-16.9 \pm 7.1$ & $-13.8 \pm 7.7$ & $0.015^{*}$ & $-22.4 \pm 14.5$ & $-19.3 \pm 12.7$ & $0.004^{* *}$ \\
\hline$\Delta$ Post-preSBP (mmHg) & $-40.3 \pm 39.3$ & $-31.8 \pm 24.8$ & $0.020^{*}$ & $-14.1 \pm 23.4$ & $-14.7 \pm 9.9$ & 0.232 & $-26.5 \pm 34.2$ & $-22.8 \pm 20.2$ & $0.011^{*}$ \\
\hline$\Delta$ Post-preDBP (mmHg) & $-12.7 \pm 10.1$ & $-12.3 \pm 10.3$ & 0.408 & $-11.0 \pm 6.3$ & $-7.8 \pm 6.3$ & $0.025^{*}$ & $-11.8 \pm 8.2$ & $-10.0 \pm 8.6$ & $0.027^{*}$ \\
\hline$\Delta$ Post-preMAP (mmHg) & $-21.9 \pm 18.6$ & $-18.8 \pm 14.9$ & $0.048^{*}$ & $-12.0 \pm 10.3$ & $-10.1 \pm 7.1$ & 0.145 & $-16.7 \pm 15.4$ & $-14.2 \pm 12.1$ & $0.014^{*}$ \\
\hline
\end{tabular}

Values are expressed as means \pm SD of the data.

Pre-: prehemodialysis; post-: posthemodialysis; SBP: systolic blood pressure; DBP: diastolic blood pressure; MAP: mean arterial pressure;

${ }^{*} P<0.05 ;{ }^{* *} P<0.01 ; \Delta$ : changes. 


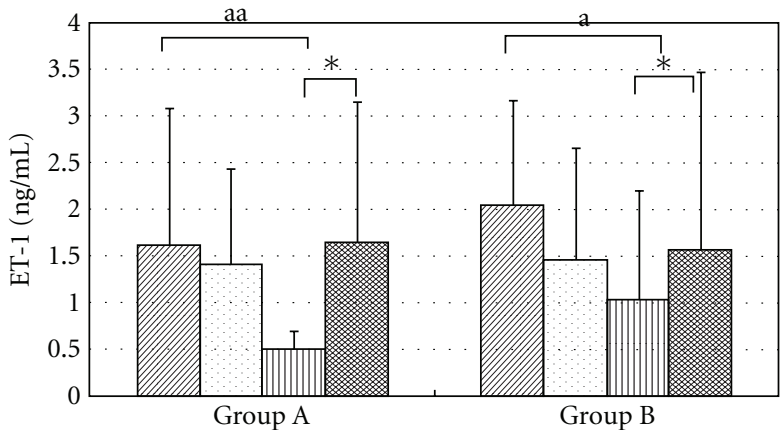

๑ Phase I predialysis

$\square$ Phase I postdialysis

国 Phase II postdialysis

(a)

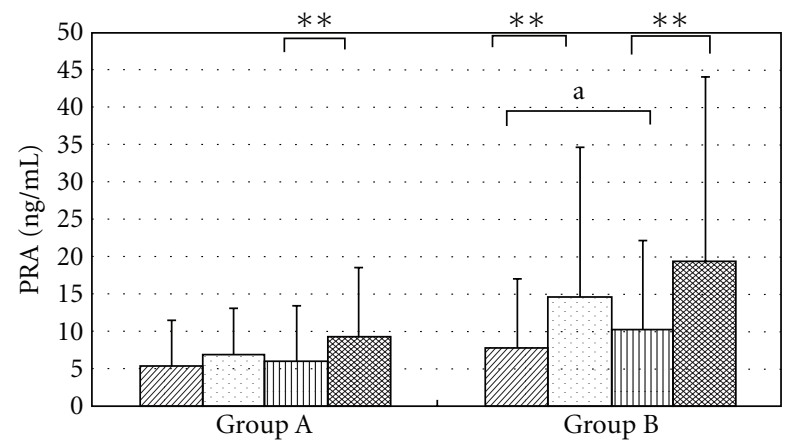

$\square$ Phase I predialysis

$\square$ Phase I postdialysis

( $)$ Phase II predialysis

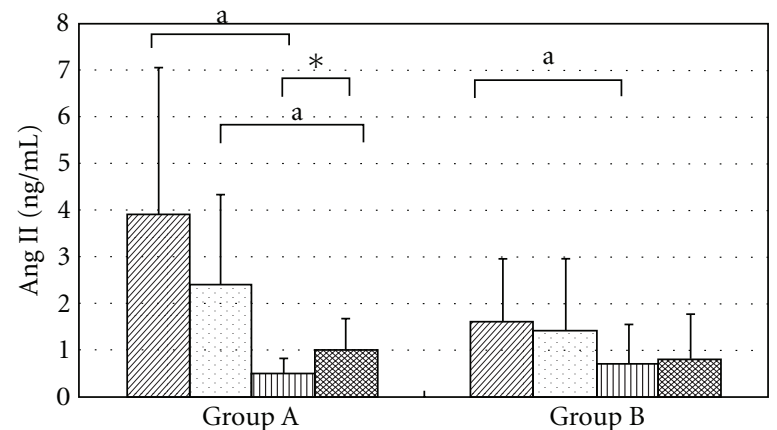

$\square$ Phase I predialysis $\quad \square$ Phase I postdialysis

四 Phase II predialysis 图 Phase II postdialysis

(b)

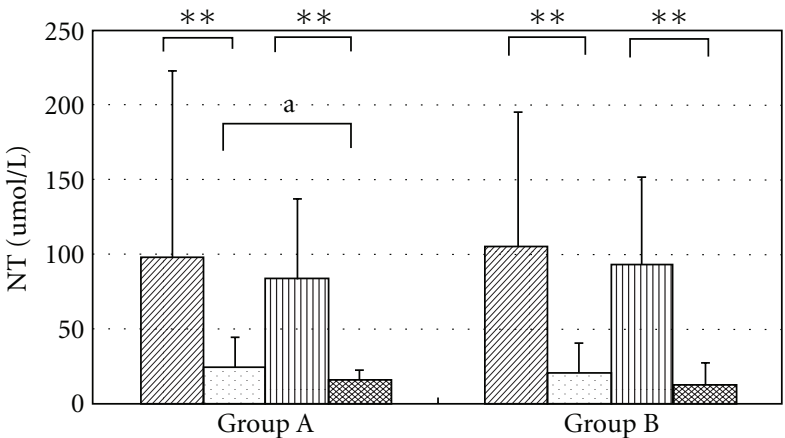

曰 Phase I predialysis $\square$ Phase I postdialysis

m Phase II predialysis $\quad$ Phase II postdialysis

(d)

FIGURE 3: Changes of endotheline-1 (ET-1), plasma renin activity (PRA), angiotensin II (AngII), and NT (Nitrate+nitrite) plasma levels after KRG treatment in patients with intradialytic hypotension. Phase I is the control phase, and phase II is the KRG treatment phase. The ET-1 levels increased significantly after hemodialysis in phase II in both group A (hypertensive at baseline, $n=18, P=0.035$ ) and group B (normotensive or hypotensive at baseline, $n=20, P=0.011$ ). The posthemodialysis PRA levels increased significantly in phase II in both group A $(P=0.005)$ and group B $(P<0.001)$. In group A, the AngII levels significantly elevated after KRG treatment $(P=0.033)$. The levels of NT decreased significantly after dialysis in all groups in both phases $(P<0.0001)$. In group A, the posthemodialysis NT level was significantly lower in phase II than those in phase I $(P=0.028) .{ }^{*} P<0.05 ;{ }^{*} P<0.01 ;{ }^{\text {a }} P<0.05$; ${ }^{\text {aa }} P<0.01$.

for the comparison of prehemodialysis AngII levels between two phases $(3.902 \pm 3.162$ to $0.477 \pm 0.353 \mathrm{ng} / \mathrm{mL}$ in group A, $P<0.001 ; 1.616 \pm 1.387$ to $0.694 \pm 0.859 \mathrm{ng} / \mathrm{mL}$ in group $\mathrm{B}, P<0.001)$. In contrast, the predialysis PRA levels remained unchanged in the group A but elevated in group B $(P=0.012)$ with KRG treatment. There were no significant changes in the prehemodialysis NT levels during the two study phases.

3.5. Side Effects. No significant adverse effects were observed during the study. Side effects reported by patients were palpitation ( $n=1$ in group A; $n=1$ in group B) and thirsty ( $n=1$ in group B). Serum potassium levels increased slightly from $4.3 \pm 0.5$ to $4.6 \pm 0.7 \mathrm{mEq} / \mathrm{L}(P=0.016)$ in group B with KRG administration, but the difference were not significant when data from both groups was pooled $(4.4 \pm 0.8$ versus $4.6 \pm 0.7 \mathrm{mEq} / \mathrm{L}, P=0.189)$ (Table 5).
Serum phosphate levels were slightly elevated from $4.9 \pm 1.4$ to $5.3 \pm 1.6 \mathrm{mg} / \mathrm{dL}(P=0.04)$ with KRG treatment, but the levels were still within the normal range. No other significant changes in hematologic or biochemical parameters were observed (Table 5).

\section{Discussion}

The current treatments for IDH include stopping ultrafiltration, increasing dialysate sodium and glucose concentrations, and administration of hypertonic solutions and vasoconstrictor agents $[1,6,12-14]$. In the current study, we found that taking $3.5 \mathrm{~g}$ KRG slices at the start of hemodialysis can elevate the nadir blood pressure and reduce the frequency of symptomatic IDH by increasing the nadir blood pressure. KRG had no significant effects on the baseline blood pressure, suggesting that its beneficial effect 
TABLE 5: Comparison of laboratory parameters with and without Korean red ginseng treatment.

\begin{tabular}{|c|c|c|c|c|c|c|c|c|c|}
\hline & \multicolumn{2}{|c|}{ Group A (HTN) } & \multirow{2}{*}{$P$ value } & \multicolumn{2}{|c|}{ Group B (non-HTN) } & \multirow{2}{*}{$P$ value } & \multicolumn{2}{|c|}{ All } & \multirow{2}{*}{$P$ value } \\
\hline & $\begin{array}{l}\text { Phase I } \\
(-\mathrm{KRG})\end{array}$ & $\begin{array}{l}\text { Phase II } \\
(+K R G)\end{array}$ & & $\begin{array}{l}\text { Phase I } \\
(- \text { KRG })\end{array}$ & $\begin{array}{l}\text { Phase II } \\
(+K R G)\end{array}$ & & $\begin{array}{l}\text { Phase I } \\
(-\mathrm{KRG})\end{array}$ & $\begin{array}{l}\text { Phase II } \\
(+K R G)\end{array}$ & \\
\hline Hct (\%) & $32.5 \pm 3.6$ & $33.2 \pm 3.3$ & 0.170 & $33.3 \pm 3.2$ & $32.9 \pm 3.6$ & 0.537 & $32.9 \pm 3.4$ & $33.0 \pm 3.4$ & 0.795 \\
\hline $\mathrm{Hb}(\mathrm{g} / \mathrm{dL})$ & $10.7 \pm 1.1$ & $10.8 \pm 1.0$ & 0.276 & $10.6 \pm 1.1$ & $10.5 \pm 1.2$ & 0.359 & $10.7 \pm 1.1$ & $10.6 \pm 1.1$ & 0.849 \\
\hline $\mathrm{Ca}(\mathrm{mg} / \mathrm{dL})$ & $9.7 \pm 1.3$ & $9.6 \pm 0.8$ & 0.938 & $10.1 \pm 1.1$ & $10.1 \pm 1.2$ & 0.618 & $9.9 \pm 1.2$ & $9.8 \pm 1.0$ & 0.543 \\
\hline $\mathrm{P}(\mathrm{mg} / \mathrm{dL})$ & $5.0 \pm 1.7$ & $5.6 \pm 1.9$ & 0.065 & $4.8 \pm 1.0$ & $5.1 \pm 1.3$ & 0.360 & $4.9 \pm 1.4$ & $5.3 \pm 1.6$ & $0.04^{*}$ \\
\hline $\mathrm{K}(\mathrm{mEq} / \mathrm{L})$ & $4.5 \pm 1.0$ & $4.5 \pm 0.7$ & 0.796 & $4.3 \pm 0.5$ & $4.6 \pm 0.7$ & $0.016^{*}$ & $4.4 \pm 0.8$ & $4.6 \pm 0.7$ & 0.189 \\
\hline $\mathrm{BUN}(\mathrm{mg} / \mathrm{dL})$ & $63.4 \pm 22.1$ & $64.3 \pm 19.2$ & 0.943 & $58.0 \pm 11.0$ & $62.2 \pm 14.9$ & 0.083 & $60.5 \pm 17.2$ & $63.2 \pm 16.9$ & 0.186 \\
\hline $\mathrm{Cr}(\mathrm{mg} / \mathrm{dL})$ & $10.1 \pm 2.9$ & $10.0 \pm 2.7$ & 0.523 & $11.3 \pm 1.9$ & $11.3 \pm 2.2$ & 0.985 & $10.7 \pm 2.4$ & $10.7 \pm 2.5$ & 0.768 \\
\hline ALT (U/L) & $16.2 \pm 10.0$ & $14.7 \pm 7.7$ & 0.459 & $15.0 \pm 6.2$ & $13.8 \pm 3.9$ & 0.599 & $15.6 \pm 8.1$ & $14.2 \pm 5.9$ & 0.183 \\
\hline
\end{tabular}

Values are expressed as means \pm SD of the data.

Hct: hematocrit; Hb: hemoglobin; Ca: calcium; P: phosphate; K: potassium. BUN: blood urea nitrogen; Cr: creatinine; ALT: alanine aminotransferase.

${ }^{*} P<0.05 ;{ }^{*} P<0.01$.

is through restoring the vasoconstrictive response to acute plasma changes during hemodialysis. Furthermore, KRG has been used in different pathologic conditions [15], and its safety profile has been well studied in patients with normal renal function [16]. Our results confirmed the safety of using oral form KRG during hemodialysis. Although KRG has been reported to increase blood pressure in non-dialysis patients [17-19, 26, 27], we did not observe exacerbation of preexisting hypertension in our patients (group A). Our results suggest that KRG may be an alternative and adjuvant treatment for IDH.

The effects of Panax ginseng on regulating blood pressure were controversial due to its complexity of major components and different actions in various pathological conditions $[18,19,22,28,29]$. It has been reported that dammarenetriol glycosides in Panax ginseng have strong CNS excitatory actions that may cause hypertension, while dammarenediol glycosides in Panax ginseng have sedative and antihypertensive effects [26]. Three decades ago, Siegel proposed that low-dose Panax ginseng could cause hypertension [18]. However, high-dose Panax ginseng was shown to increase NO production in recent clinical trials and laboratory experiments [29], which may reduce blood pressure $[28,30]$. Furthermore, Panax ginseng induced different responses in different blood vessels taken from rabbits, dogs and humans qualitatively and quantitatively [31]. Our data suggest that low-dose KRG (3.5 gm per dialysis session) could maintain the stability of blood pressure rather than exacerbating hypotension during hemodialysis.

The mechanism of IDH has been partly attributed to endothelial dysfunction in response to hemodynamic instability, with increased $\mathrm{NO}$ and decreased ET-1 during hemodialysis [4, 32-34]. ET-1 is the most potent vasoconstrictor that is locally produced from vascular endothelial cell $[35,36]$. ET-1 levels decrease in IDH prone patients and increase in patients who have hypertension during hemodialysis, implying its importance in regulating hemodynamic stability [4]. Consistently, we did not found a significant increase of ET-1 levels during hemodialysis in these hypotension-prone patients during the observation period, suggesting a lack of adequate vasoconstrictive response. Indeed, we detected a significant increase in ET1 levels during hemodialysis after KRG treatment. Similarly, we found that KRG treatment led to more activation of PRA and AngII during hemodialysis, indicating a gradually restoration of neurohormonal and cardiovascular responses to acute plasma volume change. Although NT concentrations is an indirect measurement of $\mathrm{NO}$ and can be removed by dialysis [4], we observed a significant decrease in the posthemodialysis NT levels after KRG treatment (group A). The result suggested that the beneficial effects of KRG treatment on IDH may be partially due to decreased NO production.

Previous studies have shown that hypotension-prone patients have higher baseline AngII levels compared to hypotension resistant dialysis patients [37]. This indicates that renin-angiotensin-aldosterone system may be abnormally activated in patients with recurrent IDH but still incapable to have adequate cardiovascular capacitance to the dialytic ultrafiltration. Elevation of AngII may lead to endothelial dysfunction and increase the risk of adverse cardiovascular events [38]. In the current study, we found that KRG treatment resulted in significantly reduced baseline AngII and ET-1. Our data suggest that KRG may have additional beneficial effects on endothelial dysfunction in patients with IDH by lowering the baseline of ET- 1 and AngII levels [36, 39].

Despite the promising results obtained in this trial, several limitations should be acknowledged. First, the study had a relative small sample size, and further studies are 
needed to confirm the results. Second, the study is a phase I pilot study, and we could only observe the differences within the same patient although this also help to eliminate the variation of blood pressure changes among different patients. Third, the long-term effects of KRG in hemodialysis patients were not studied, and further investigations are warranted.

\section{Conclusions}

Chewing low-dose KRG renders patients better resistance to acute $\mathrm{BP}$ reduction during hemodialysis. KRG treatment improves the compensatory response to acute volume change during hemodialysis via activation of vasoconstrictors (ET1 and AngII). Our results suggest that KRG could be an adjuvant treatment for IDH.

\section{Acknowledgments}

This study is supported by a Grant from Chang Gung Memorial Hospital (CMRPG380321). The authors' sincere thanks are due to Redox Functional Medicine Laboratory and dialysis staff at hemodialysis center in Taoyuan Chang Gung Memorial Hospital (TCGMH) for the provision of excellent technical assistance.

\section{References}

[1] B. F. Palmer and W. L. Henrich, "Recent advances in the prevention and management of intradialytic hypotension," Journal of the American Society of Nephrology, vol. 19, no. 1, pp. 8-11, 2008.

[2] A. Tislér, K. Akócsi, B. Borbás et al., "The effect of frequent or occasional dialysis-associated hypotension on survival of patients on maintenance haemodialysis," Nephrology Dialysis Transplantation, vol. 18, no. 12, pp. 2601-2605, 2003.

[3] J. T. Daugirdas, "Pathophysiology of dialysis hypotension: an update," American Journal of Kidney Diseases, vol. 38, no. 4, pp. S11-S17, 2001.

[4] D. S. C. Raj, B. Vincent, K. Simpson et al., "Hemodynamic changes during hemodialysis: role of nitric oxide and endothelin," Kidney International, vol. 61, no. 2, pp. 697-704, 2002.

[5] M. A. Perazella, M. Rho, and U. C. Brewster, "Vasopressin insufficiency and intradialytic hypotension," American Journal of Kidney Diseases, vol. 52, no. 6, p. 1197, 2008.

[6] F. M. van der Sande, G. Wystrychowski, J. P. Kooman et al., "Control of core temperature and blood pressure stability during hemodialysis," Clinical Journal of the American Society of Nephrology, vol. 4, no. 1, pp. 93-98, 2009.

[7] E. Movilli, C. Camerini, B. F. Viola, N. Bossini, A. Strada, and R. Maiorca, "Blood volume changes during three different profiles of dialysate sodium variation with similar intradialytic sodium balances in chronic hemodialyzed patients," American Journal of Kidney Diseases, vol. 30, no. 1, pp. 58-63, 1997.

[8] N. R. Skroeder, S. H. Jacobson, L. E. Lins, and C. M. Kjellstrand, "Acute symptoms during and between hemodialysis: the relative role of speed, duration, and biocompatibility of dialysis," Artificial Organs, vol. 18, no. 12, pp. 880-887, 1994.

[9] G. Rostoker, M. Griuncelli, C. Loridon, A. Benmaadi, and E. Illouz, "Left-ventricular diastolic dysfunction as a risk factor for dialytic hypotension," Cardiology, vol. 114, no. 2, pp. 142$149,2009$.
[10] M. H. Chang and K. J. Chou, "The role of autonomic neuropathy in the genesis of intradialytic hypotension," American Journal of Nephrology, vol. 21, no. 5, pp. 357-361, 2001.

[11] D. Sapoznikov, R. Backenroth, and D. Rubinger, "Baroreflex sensitivity and sympatho-vagal balance during intradialytic hypotensive episodes," Journal of Hypertension, vol. 28, no. 2, pp. 314-324, 2010.

[12] M. M. Barakat, Z. M. Nawab, A. W. Yu, A. H. Lau, T. S. Ing, and J. T. Daugirdas, "Hemodynamic effects of intradialytic food ingestion and the effects of caffeine," Journal of the American Society of Nephrology, vol. 3, no. 11, pp. 1813-1818, 1993.

[13] J. H. Song, G. H. Park, S. Y. Lee, S. W. Lee, S. W. Lee, and M. J. Kim, "Effect of sodium balance and the combination of ultrafiltration profile during sodium profiling hemodialysis on the maintenance of the quality of dialysis and sodium and fluid balances," Journal of the American Society of Nephrology, vol. 16, no. 1, pp. 237-246, 2005.

[14] D. N. Cruz, R. L. Mahnensmith, H. M. Brickel, and M. A. Perazella, "Midodrine is effective and safe therapy for intradialytic hypotension over 8 months of follow-up," Clinical Nephrology, vol. 50, no. 2, pp. 101-107, 1998.

[15] Y. Z. Xiang, H. C. Shang, X. M. Gao, and B. L. Zhang, "A comparison of the ancient use of ginseng in traditional Chinese medicine with modern pharmacological experiments and clinical trials," Phytotherapy Research, vol. 22, no. 7, pp. 851-858, 2008.

[16] J. T. Coon and E. Ernst, "Panax ginseng: a systematic review of adverse effects and drug interactions," Drug Safety, vol. 25, no. 5, pp. 323-344, 2002.

[17] M. S. Bahrke and W. P. Morgan, "Evaluation of the ergogenic properties of ginseng: an update," Sports Medicine, vol. 29, no. 2, pp. 113-133, 2000.

[18] R. K. Siegel, "Ginseng and high blood pressure," Journal of the American Medical Association, vol. 243, no. 1, p. 32, 1980.

[19] UptoDate, "Panax gnseng: natural drug information".

[20] Y. S. Keum, K. K. Park, J. M. Lee et al., "Antioxidant and antitumor promoting activities of the methanol extract of heatprocessed ginseng," Cancer Letters, vol. 150, no. 1, pp. 41-48, 2000.

[21] W. Y. Kim, J. M. Kim, S. B. Han et al., "Steaming of ginseng at high temperature enhances biological activity," Journal of Natural Products, vol. 63, no. 12, pp. 1702-1704, 2000.

[22] K. T. Choi, "Botanical characteristics, pharmacological effects and medicinal components of Korean Panax ginseng C A Meyer," Acta Pharmacologica Sinica, vol. 29, no. 9, pp. 11091118, 2008.

[23] National Kidney Foundation, "K/DOQI Clinical Practice Guidelines for cardiovascular disease in dialysis patients," American Journal of Kidney Diseases, vol. 45, supplement 4, pp. S49-S59, 2005, pp. S69-S75.

[24] S. Prakash, A. X. Garg, A. P. Heidenheim, and A. A. House, "Midodrine appears to be safe and effective for dialysisinduced hypotension: a systematic review," Nephrology Dialysis Transplantation, vol. 19, no. 10, pp. 2553-2558, 2004.

[25] N. Ky, "The comparative understanding between red ginseng and white ginsengs, processed ginsengs (Panax ginseng C. A. Meyer)," Journal of Ginseng Research, vol. 1, no. 1, pp. 1-18, 2005.

[26] R. K. Siegel, "Ginseng abuse syndrome. Problems with the panacea," Journal of the American Medical Association, vol. 241, no. 15, pp. 1614-1615, 1979.

[27] M. S. Bahrke, W. P. Morgan, and A. Stegner, "Is ginseng an ergogenic aid?" International Journal of Sport Nutrition and Exercise Metabolism, vol. 19, no. 3, pp. 298-322, 2009. 
[28] K. H. Han, S. C. Choe, H. S. Kim et al., "Effect of red ginseng on blood pressure in patients with essential hypertension and white coat hypertension," American Journal of Chinese Medicine, vol. 26, no. 2, pp. 199-209, 1998.

[29] B. H. Jeon, C. S. Kim, H. S. Kim, J. B. Park, K. Y. Nam, and S. J. Chang, "Effect of Korean red ginseng on blood pressure and nitric oxide production," Acta Pharmacologica Sinica, vol. 21, no. 12, pp. 1095-1100, 2000.

[30] M. F. Caron, A. L. Hotsko, S. Robertson, L. Mandybur, J. Kluger, and C. M. White, "Electrocardiographic and hemodynamic effects of Panax ginseng," Annals of Pharmacotherapy, vol. 36, no. 5, pp. 758-763, 2002.

[31] X. Chen, C. N. Gillis, and R. Moalli, "Vascular effects of ginsenosides in vitro," British Journal of Pharmacology, vol. 82, no. 2, pp. 485-491, 1984.

[32] E. M. El-Shafey, G. F. El-Nagar, M. F. Selim, H. A. El-Sorogy, and A. A. Sabry, "Is there a role for endothelin-1 in the hemodynamic changes during hemodialysis?" Clinical and Experimental Nephrology, vol. 12, no. 5, pp. 370-375, 2008.

[33] K. Yokokawa, M. Kohno, and J. Yoshikawa, "Nitric oxide mediates the cardiovascular instability of haemodialysis patients," Current Opinion in Nephrology and Hypertension, vol. 5, no. 4, pp. 359-363, 1996.

[34] K. Yokokawa, R. Mankus, M. G. Saklayen et al., "Increased nitric oxide production in patients with hypotension during hemodialysis," Annals of Internal Medicine, vol. 123, no. 1, pp. 35-37, 1995.

[35] W. G. Haynes and D. J. Webb, "Contribution of endogenous generation of endothelin-1 to basal vascular tone," The Lancet, vol. 344, no. 8926, pp. 852-854, 1994.

[36] H. Liu, Y. Peng, F. Liu et al., "Correlation between endothelin1 and atherosclerosis in chronic hemodialysis patients," Journal of Nephrology, vol. 23, no. 5, pp. 593-602, 2010.

[37] G. Graziani, S. Finazzi, R. Mangiarotti et al., "Different cardiovascular responses to hemodialysis-induced fluid depletion and blood pressure compliance," Journal of Nephrology, vol. 23, no. 1, pp. 55-61, 2010.

[38] E. L. Schiffrin, M. L. Lipman, and J. F. E. Mann, "Chronic kidney disease: effects on the cardiovascular system," Circulation, vol. 116, no. 1, pp. 85-97, 2007.

[39] J. Sung, K. H. Han, J. H. Zo, H. J. Park, C. H. Kim, and B. H. Oh, "Effects of red ginseng upon vascular endothelial function in patients with essential hypertension," American Journal of Chinese Medicine, vol. 28, no. 2, pp. 205-216, 2000. 


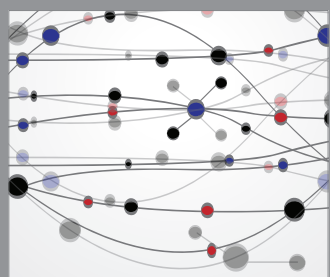

The Scientific World Journal
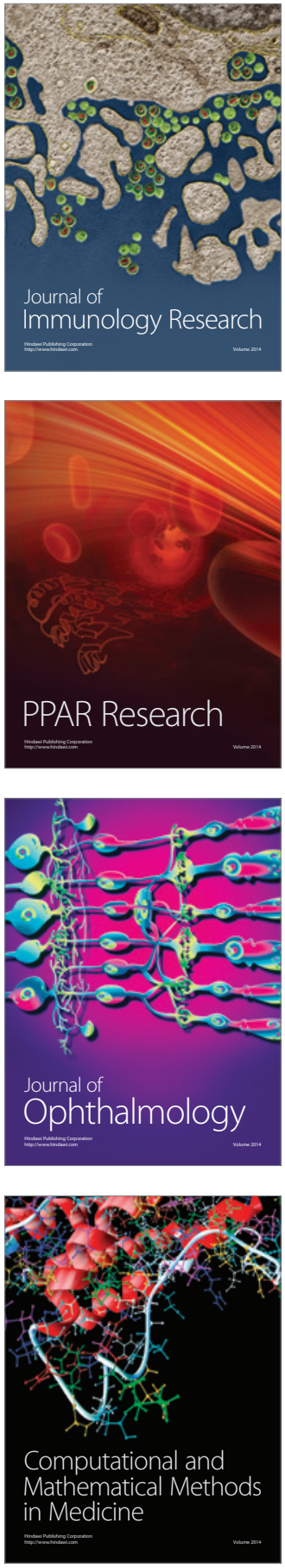

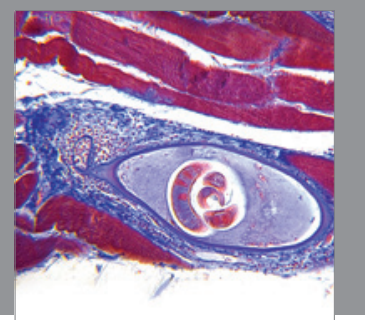

Gastroenterology

Research and Practice
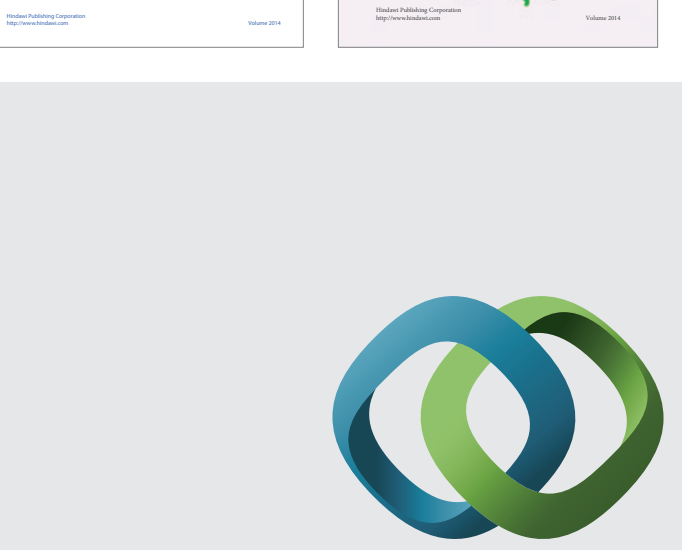

\section{Hindawi}

Submit your manuscripts at

http://www.hindawi.com
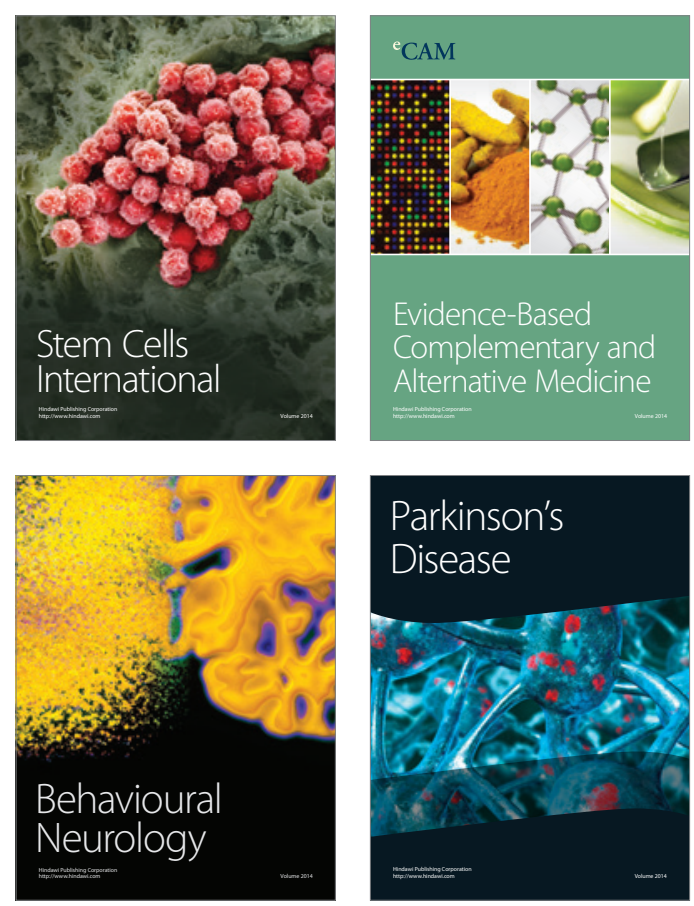

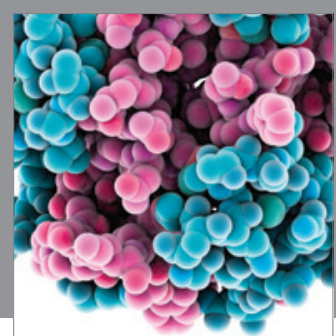

Journal of
Diabetes Research

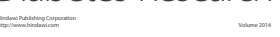

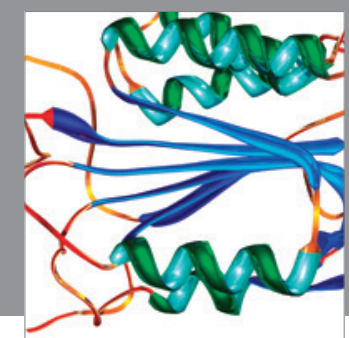

Disease Markers
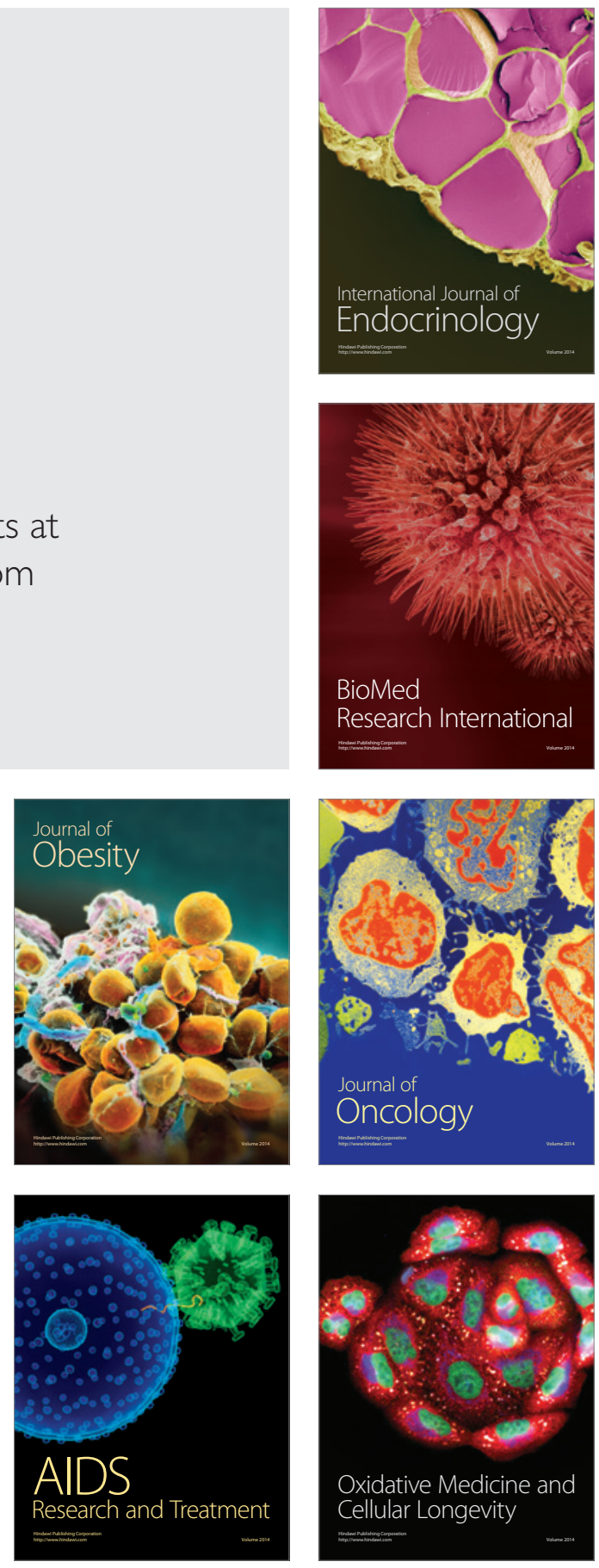